90. From the anxious expression on her face, the sudden onset of the pain, and its persistent character, I made a tenta tive diagnosis of appendicitis. I was called again at 11 A.M. and found the pain more aggravated and somewhat localized in the right iliac region. Moderate doses of morphin had not abated the suffering in the least. The bowels had been freely moved in the meantime by magnesia citrate. Her temperature at this hour was 100 degrees F.; pulse 110 . I told the nurse to continue the morphin and apply hot applications to the abdomen.

At 2 P.M. I saw her in consultation with Arthur R. Edwards, M.D., and found her temperature 101.5 degrees F., pulse 120, with an area of tenderness over the lower and right half of the abdomen.

A positive diagnosis of appenditis was made and immediate operation advised. She was removed to St. Luke's Hospital and operated on at 4 P.M. The usual incision was made and on opening the abdomen, a large quantity of peritoneal fluid escaped. The peritoneal gloss was absent from the intestines for a considerable area, demonstrating conclusively the existence of peritonitis of considerable intensity. The appendix, readily brought in to the wound, was to all appearances perfectly healthy. On examination, however, bulbosity could be felt at its distal extremity. For this reason I decided to amputate it. After removal, it was cut open and found to contain a small quantity of fecal matter, together with a live ascaris lumbricoides. The superficial epigastric artery crossed the abdomen very high up, and gave rise to troublesome hemorrhage before it could be secured.

This patient did nicely for about thirty six hours, when her pulse began to fail. It gradually became weaker and increased in frequency in spite of free stimulation, till Tuesday afternoon, when it was 160 per minute and very small. The respirations were sighing and labored, with great restlessness. At this time eight ounces of a normal salt solution was injected hypodermatically. A good result was immediate, and from that time on the patient made an uninterrupted recovery.

In Case 1 on examination of the appendix after its removal, a depression was marked at the cecal opening. Evidently a hard fecal mass had become lodged there, making a pressure on the sclerosed appendiceal blood vessels, and in this way causing gangrene. The appendix was nicely shut off from the general cavity by layers of plastic lymph, agglutinating the cecum and ileum. Perforation was inevitable with the formation of an abscess cavity. In this instance an early operation saved the patient weeks of invalidism, and the increased danger of a fatal result from abscess rupturing into the abdominal cavity.

Case 2 is the first instance I have been able to find of a lubricoid causing appendicitis. The worm was lying in the lumen of the appendix and, to all appearances, had not perforated its wall. The surrounding peritonitis was intense and leads me to advance the theory of a toxin being generated by the worm, which was transmitted to the peritoneum by osmosis, thus setting up the chain of symptoms. On the other hand the worm may have penetrated the wall of the appendix and infected the peritoneum by contact.

In both these cases very small doses of morphia sul. phate were given to control pain, but not sufficient to check peristalsis, or mask the symptoms.

In the first instance the patient was made comfortable; in the second, any dose of the opiate I felt it was safe to administer did not relieve the agony from which the patient suffered.

189 41st Street.

\section{THE PREPARATION AND TESTING OF DIPHTHERIA ANTITOXIN}

BY GEORGE W. COX, M.D. CHICAGO.

In a recent report to the Michigan State Board of Health, Dr. Charles T. McClintock of Detroit, makes the statement that "inspection of the most important laboratories of Germany, France, Austria and Great Britain reveals the fact that the methods in use for the production of diphtheria antitoxic serum and vaccine are much the same everywhere," etc. While this statement is entirely correct in a general way, it carries with it the impression that if the same methods are used by the various manufacturers, one could make just as good a serum as another, an impression as dangerous as it is erroneous. It is only necessary to mention the great discrepancy that exists between many of our drugs of the same name in order to illustrate this point. Essentially the same methods are used by all our manufacturers in the production of fluid extracts, for example, and yet our markets are burdened with every grade and shade of these remedies from the absolutely perfect to the absolutely worthless.

There are many reasons for this, the most conspicuous being a lack of care in the selection and assaying of the crude material; the use of a poor menstruum; too great haste in the preparation, and the employment of unskilled or inexperienced labor. If, then, we notice such a variation in the quality of the remedies just mentioned, how much more we might reasonably expect to find it in such delicate substances as antitoxic blood serums, where so much more skill, experience and patience are required in their preparation. Dr. McClintock tells us that in Germany any one may manufacture antitoxin, but before it is offered for sale it must be tested by a government official. In the United States we go Germany one better. We not only allow anybody to manufacture antitoxin, but we allow anybody to test it, certify to its strength and sell it. In the report above alluded to, mention is made of dividing a bottle of serum into four equal parts and sending them to four different bacteriologists for the purpose of testing. One reported the strength to be 100 units per c.c., one 150 units, one 175 units and the fourth 250 units per c.c. Aside from the amusing aspect of such extravagant variation, it would be interesting to know how that lot of serum was labeled when it was put upon the market. Who does the testing of our domestic serums, any. way? We know that in Germany they are tested by Prof. Ehrlich, who was appointed by the government for that purpose, and we have every reason to believe that his work is uniform and reliable. In France the manufacture of antitoxin is still more directly under government control. Two or three institutions in that Republic have been authorized by special ministerial decree to make and test antitoxin, the two principal ones being the great Pasteur Laboratories at Paris and Lille, under the direction of Prof. Roux and Dr. Calmette, respectively. Inasmuch as we must rely upon the name and reputation of the manufacturer for our guidance in the selection of a serum for use, it seems but the part of wisdom for us to lay aside all prejudice and sentimentality and choose one whose label bears a name known and honored throughout the world. Diphtheria is such a treacherous and fatal disease, and our responsibility in combating it so grave, that we can not afford to jeopardize our own reputation, to say nothing about the life of the patient, by using a serum that is not vouched for by someone whose authority is recognized everywhere. In this connection it may be stated that there are four names so intimately associated with diphtheria and its cure, that the mere mention of the disease at once suggests these names: Roux, the discoverer of the diphtheria 
toxin; Behring, the discoverer of the antitoxin; Calmette, the distinguished director of the Pasteur Laboratories at Lille, and Ehrlich who created the world's standard for the testing of serums. The first mentioned of this group, whose great discovery made it possible for the others to follow with their specialties, is still in the harness at the Pasteur Laboratories in Paris, exercising personal supervision over every branch of the preparation of the serum that bears his name. He not only sees that the finished products are properly tested and sealed, but he makes sure that the tests are made with toxins of the proper degree of virulence. He also prepares his serums without antiseptics, having proved not only that such antiseptics are prejudicial to the patient, but that the serums retain their freshness and potency longer without these extraneous substances than they do with them. In these two very important respects, then, it must be said that Roux's methods are not used 'almost everywhere," nor indeed almost anywhere, except in his own laboratory. Much has been said about the relative antitoxic strength of different serums, and some fabulous claims have been made in this direction. Roux has never been favorably inclined toward serums of extra high putency, say 400 or 500 units per c.c., for the simple reason that while it is easy enough to produce them, they do not retain their strength as well as those of lesser potency, 150 to 300 units per c.c.; and if antiseptics are added, the deterioration is all the more pronounced and rapid. Originally Roux's serums were quite weak as compared with the strengths claimed for others, and consequently large doses were required; but he has gradually increased the strength until his standard serum is now about three times as strong as it was eighteen months ago. That is to say, his "regular" serum contains 150 units per c.c., while the "extra" prepared by Calmette, is just double that strength. Roux has satisfied himself, by long-continued experimentation, that this last named strength ( 300 units per c.c.) is about the limit beyond which a serum can not be depended upon to retain its full potency for any length of time. Inasmuch as there is no more danger in administering these antitoxins than there would be in using pure blood serum, the dosage is practically unlimited, and the required antitoxic power may always be given without fear, regardless of the bulk. However, in response to the clamor for a serum of a higher antitoxic power, Roux has now entrusted Calmette with the preparation of one of at least 500 units per c.c. This is known as his "Concentrated" serum, and is dispensed in vials of 5 c.c. or 2500 units.

It is to be hoped that my readers will pardon me for thus dilating upon the excellencies of any one antitoxin, especially one of foreign manufacture, as sentiments of loyalty should and do naturally incline one's preference toward a home product. But aside from the great respect that is due from the entire world to the pathfinder in bacteriologic science, I am in a position to know what is going on at the Pasteur Laboratories at all times, and I do not allow my enthusiasm to lead me into statements that can not be backed up by authority from headquarters. It seems to me more than likely that if Dr. McClintock had given the Pasteur Laboratories a thorough inspection, he would not only have met there a full half dozen of the world's greatest bacteriologic specialists, but he would also have noticed many details in the methods of preparing and testing antitoxins not to be seen elsewhere. For example, he would have noticed that they do not prepare their serums in a hurry. Nature having a considerable part in this process, she takes her own time in its performance, as she does in all her works, and no effort is made to abbreviate it. One particular serum that requires twelve months for its production by its discoverer is made in this country in less than half that time. It is not necessary to make comparisons as to the quality of the two products. The doctor would also have observed that the 300 or 400 horses from which the various serums are taken are kept in the country, many miles from the unsanitary surroundings of city stables, and that a portion of the time during treatment is spent in green pastures where nature's food, pure air and voluntary exercise are enjoyed to the full. It must be confessed that these and many other details in the production of antitoxin at the Pasteur Laboratories are at great variance with those in vogue at other places, and not altogether to the credit of the "other places." Finally, the writer has always been of the opinion that the laxity of our laws upon sanitation, is a mark of shame. ful carelessness on the part of Congress, a disgrace to the members of the medical profession for silently submitting to legislative inactivity and a constant menace to the welfare of our people. The testing of antitoxins should be under government control. Are we not as worthy of such protection as the French or Germans? I think so; and as a starter in this direction I move that the profession as a body appeal to Congress for the establishment of a Department of Public Health.

2258 Wabash Avenue.

\section{ALKALIN ANTISEPTIC TABLETS. BY HENRY W. WANDLESS, M.D. DALLAS, TEXAS.}

Each tablet is composed of the following: Acid boracic, grs. 22.5; sodii bicarb., grs. 7.5; sodii biborat., grs. 7.5; camphor, grs. 17; menthol, grs. 17 ; thymol, gr. .5 combined with three minims of the following combinations of oils: Oil pinus pinolias, 2 parts; oil eucalyptus, 2 parts; oil Scotch pine, 2 parts; oil spruce, 1 part; oil cedar, 1 part; oil cubebs, 1 part; oil wintergreen, .25 part; oil bay leaves .25 part; chloroform, 2 parts.

These tablets are very useful in the treatment of catarrhal conditions of the nose, throat and ear. They are used by dissolving them in hot water and used as a douche or spray, and are sometimes useful dissolved in the mouth in cases of sore throat and troublesome cough caused by irritation of the larynx. One of these tablets dissolved in one or two ounces of boiling water will make a very agreeable wash or spray for the nose, throat or ear. I usually have made one gallon of the solution at one time, which is prepared as follows: after the water reaches the boiling point, one tablet is added for each fluid ounce and allowed to boil for five minutes, after which the solution is placed in a macerating jar and allowed to stand with occasional shaking, from three to four weeks, or until needed for use. It is then filtered or siphoned from the bottom without disturbing the top and is then ready for use. The solution, prepared as described, imparts a very rich, mellow odor; and while it is a little sharp when first sprayed into the nose this is followed by a delightfully refreshing and soothing effect.

The efficacy of the solution does not seem to be 